\title{
Association between single nucleotide polymorphisms rs72525532, rs45596738, rs148759216, rs188133936, and rs114627122 of APOA5 gene in children and adolescents with metabolic syndrome
}

\author{
Samaneh Salehi $^{1}$, Modjtaba Emadi-Baygi ${ }^{1,2^{*}}{ }^{\mathbb{D}}$, Parvaneh Nikpour $^{3,4}$, Roya Kelishadi $^{4}$ \\ 'Department of Genetics, Faculty of Basic Sciences, Shahrekord University, Shahrekord, Iran \\ ${ }^{2}$ Research Institute of Biotechnology, Shahrekord University, Shahrekord, Iran \\ ${ }^{3}$ Department of Genetics and Molecular Biology, Faculty of Medicine, Isfahan University of Medical Sciences, Isfahan, Iran \\ ${ }^{4}$ Child Growth and Development Research Center, Research Institute for Primordial Prevention of Non-communicable Disease, \\ Isfahan University of Medical Sciences, Isfahan, Iran
}

*Corresponding Author: Modjtaba Emadi-Baygi, Department of Genetics, Faculty of Basic Sciences, Shahrekord University, Shahrekord, Iran. Tel: +98 38 32324419, Fax: +98 38 32324419, Email: emadi-m@sci.sku.ac.ir

\begin{abstract}
Background and aims: The APOA5 gene is one of the genes involved in metabolic syndrome (MetS), as a constellation of several cardiovascular disease (CVD) risk factors. The present study evaluated the possible associations between five single nucleotide polymorphisms (SNPs) in the microRNA target site (miR-TS-SNPs) of the APOA5 gene with MetS.

Methods: This case-control study included 57 MetS cases, along with 59 normal children and adolescents aged 9-18 years. All miR-TSSNPs rs188133936, rs72525532, rs45596738, rs148759216, and rs114627122 were genotyped by polymerase chain reaction-sequencing. Independent t-test, as well as the chi-square test and logistic regression analysis was used to determine the association of SNPs with MetS risk and its clinical components.

Results: The mean (SD) age of MetS participants and controls was $12.35(0.25)$ and $13.39(0.38)$ years, respectively. Although no nucleotide changes were present in rs188133936, rs45596738, rs148759216, and rs114627122, a greater frequency of A insertion was detected in rs72525532 in MetS cases compared with the control group $(P=0.012)$. This variant showed a significant difference in triglycerides (TG) and high-density lipoprotein cholesterol (HDL) levels between different genotype groups $(P<0.0001$ and $P=0.05$, respectively) in controls. Furthermore, AA insertion genotype was correlated with an increased risk of MetS (Odds ratio $[95 \% \mathrm{Cl}]=8.12[0.966-68.27], P=0.05$ ). Conclusion: This study was the first to investigate the association between rs188133936, rs45596738, rs148759216, rs76463524, and rs72525532 variants of the APOA5 gene and MetS. Our findings reveal that rs72525532 might have an impact on TG, HDL levels, and the risk of MetS.

Keywords: Metabolic syndrome, APOA5, Single nucleotide polymorphism, miRNA
\end{abstract}

Received: 17 June 2017, Accepted: 36 January 2019, ePublished: 30 August 2019

\section{Introduction}

Metabolic syndrome (MetS), or the insulin resistance syndrome (syndrome $\mathrm{X}$ ), is considered as a major public health problem that is the clustering of clinical features including elevated blood pressure, abdominal obesity, insulin resistance, and dyslipidemia. In addition, MetS is recognized as an independent risk factor for type 2 diabetes, cardiovascular diseases (CVD), and CVD mortality (13 ). Based on the result of some studies, this syndrome is prevalent among the Iranian population $(4,5)$. Similarly, MetS has an intricate etiology and represents the results from the interactions of genetics and environmental factors (6). Hyperlipidemia refers to any disease resulting in high levels of plasma triglyceride (TG), cholesterol and low-density lipoprotein, and low levels of highdensity lipoprotein cholesterol (HDL-C) concentrations. As the risk factor for CVD, various genes, including apolipoprotein A5 (APOA5) located on chromosome $11 \mathrm{q} 23$ in the APOA1-C3-A4 gene cluster $(7,8)$, are involved in the pathology of hyperlipidemia. This gene expresses the APOAV protein, which is synthesized in the liver. Further, APOAV protein is believed to increase the levels of plasma TG while reducing the HDL-C levels and interacting with lipoprotein lipase $(9,10)$. MicroRNAs (miRNAs) are a group of non-protein-coding RNAs which are demonstrated to play important regulatory roles in diverse biological processes like adiposity, insulin resistance, and appetite regulation (11), as well as having

(C) 2019 The Author(s); Published by Shahrekord University of Medical Sciences. This is an open-access article distributed under the terms of the Creative Commons Attribution License (http://creativecommons.org/licenses/by/4.0), which permits unrestricted use, distribution, and reproduction in any medium, provided the original work is properly cited. 
a critical role in metabolism (12). MiRNAs also create a perfect Watson-Crick match by binding to nucleotides 2-8 from the 5' end of the miRNA (the seed sequence) to their target sequences in the 3' untranslated region of mRNAs. By binding to the target site, miRNAs inhibit translation and induce cleavage/decay of their target mRNAs (1214). Single nucleotide polymorphisms (SNPs) in miRNA gene (miR-SNPs) or miRNAs target site (miR-TS-SNP) probably affect the gene expression levels (13-15).

Likewise, miR-TS-SNP can disturb miRNA target sites or create a new miRNA binding site and have a tremendous effect on diverse biological functions, including disease susceptibilities, cancers, Parkinson's disease, osteoporosis, diabetes, hypertension, and MetS $(16,17)$. Several variants (miR-TS-SNPs) have been identified in the APOA5 gene, including rs72525532 (c.*285_*286insGA), rs188133936 (c.*191 C>T), rs114627122 (c. ${ }^{*} 172$ C>T), rs45596738 (c. ${ }^{*} 288-289$ insGA), and rs148759216 (c. ${ }^{*} 289-290$ insAG) (18). These polymorphisms occur in miRNA binding site on mRNA that leads to the gain or loss of the binding site for these miRNAs. So far, no study has examined the association of these five miR-TS-SNPs of $A P O A 5$ gene, along with the risk of MetS and its major components in children and adolescents. Therefore, the current study aimed to evaluate the genotype and allele distributions of these variants in a cohort of MetS children and adolescent to assess if there is an association between such variants and the risk of MetS.

\section{Materials and Methods}

This report is a case-control study which contained 116 children and adolescents from Isfahan, a central province in Iran. The case group included $57 \mathrm{Met} S$ children diagnosed with modified ATP III (Adult Treatment panel III) criteria as follows:

- Waist circumference $>75$ th percentile for the age and gender in the studied population;

- $\quad$ Fasting TG $\geq 100 \mathrm{mg} / \mathrm{dL}$;

- $\quad$ Serum HDL-C<50 mg/dL;

- $\quad$ Systolic blood pressure/diastolic blood pressure $>90$ th percentile for gender, age, and height fasting blood sugar $\geq 100 \mathrm{mg} / \mathrm{dL}$.

Further, MetS was defined as the presence of at least three of the above-mentioned metabolic traits (19). Fiftynine control subjects had no clinical and laboratory marker of MetS, diabetes, or cardiovascular disorders.

MiRNAs targeting variations within apolipoprotein A5 (APOA5) target gene, which lead to the gain or loss of the binding site for these miRNAs, were identified by using miRNA-related SNP (20), PolymiRTS (21), and MirSNP databases (22). Furthermore, TargetScan (23), Mirwalk (24), and the miRanda (25) databases were utilized to predict miRNA target sites within the 3' untranslated regions of the APOA5 gene (Table 1 ).

Then, DNA was extracted from blood leukocytes by using the Diatome kit (Isogen Laboratory, Russia). The amplification and genotyping of all SNPs at the APOA5 gene were performed by applying polymerase chain reaction (PCR) and Sanger sequencing methods.

Similarly, primers A5 forward (5'AGGCACTGGGACTGAGGAAG -3') and A5 reverse (5'-GGCAGCCAGAAGTGACTAGAG-3') were designed for the amplification of the $708 \mathrm{bp}$ regions containing these SNPs. Moreover, to amplify the desired segment, the PCR conditions were followed as initial denaturation at $95^{\circ} \mathrm{C}$ for 2.5 minutes, 35 cycles of denaturation at $95^{\circ} \mathrm{C}$ for 50 seconds, annealing at $62^{\circ} \mathrm{C}$ for 70 seconds, extension at $72^{\circ} \mathrm{C}$ for 70 seconds, and the final extension at $72^{\circ} \mathrm{C}$ for 15 minutes. PCR reagent mixture included $0.25 \mu \mathrm{L}$ of Taq DNA polymerase $(5 \mathrm{U} / \mu \mathrm{L}, \mathrm{KBC}$, Tehran, Iran), $10 \mathrm{X}$ buffer $(2.5 \mu \mathrm{L}), 0.75 \mu \mathrm{L} \mathrm{MgCl}_{2}(50$ $\mathrm{mM}), 0.5 \mu \mathrm{L}$ deoxynucleoside triphosphate $(40 \mathrm{mM}), 2$ $\mu \mathrm{L}$ DNA $(200 \mathrm{ng} / \mu \mathrm{L}), 0.5 \mu \mathrm{L}$ appropriate specific primer pairs $(10 \mathrm{pmol} / \mu \mathrm{L})$, and water in the final volume of 25 $\mu \mathrm{L}$. The genotypic distribution between the cases and controls were compared by the Chi-square test, followed by an independent $t$ test to compare the clinical conditions of MetS between all the genotypes of rs72525532. The association between the genotypes and MetS risk was estimated by simple and multivariate logistic regressions through calculating the odds ratios (ORs) and 95\% confidence intervals (CIs). Finally, statistical analyses were performed using SPSS software, version 16.0 (SPSS, Inc., Chicago, IL). All data were expressed as mean \pm standard error of mean and $P$ values lower than 0.05 were considered statistically significant.

\section{Results}

This study included 57 MetS participants (children and adolescents) with the mean (SD) age of 12.35 (0.25) years and 59 controls with the mean (SD) age of $13.39(0.38)$ years. Table 2 represents the clinical characteristics of MetS individual and control subjects, including plasma TG, total cholesterol (TC), low-density lipoprotein-cholesterol (LDL-C), high-density lipoprotein-cholesterol (HDL-C) levels, and body mass index.

As expected, the patient subjects had significantly higher TG, TC, LDL-C levels, and body mass index $(P<0.0001)$, and lower levels of HDL-C $(P<0.0001)$. The result of genotyping of four SNPss at apolipoprotein A5 (APOA5) gene, including rs188133936 (c.*191 C>T), rs114627122 (c. ${ }^{*} 172$ C>T), rs45596738 (c.*288-289 insGA), and rs148759216 (c.*289-290 insAG) represented no changes, while an insertion A was found at the $3^{\prime}$ untranslated region (UTR) position 285-286 in rs72525532 (c.*285286 insGA).

Based on the data obtained from the NCBI, the rs72525532 was reported as the insertion GA in position 285-286 at 3' UTR of the APOA5 gene. The genotype distributions of this variant between 116 selected 
Table 1. The list of miR-TS-SNPsof APOA5 gene

\begin{tabular}{|c|c|c|c|}
\hline Polymorphism & Allele & microRNA & Algorithm \\
\hline \multirow{2}{*}{ rs114627122 } & $\mathrm{T}$ & hsa-miR-513a-5p & \multirow{2}{*}{ miRNASNP, PolymiRTS Database, MirSNP, and TargetScan } \\
\hline & $\mathrm{C}$ & hsa-miR-4722-5p & \\
\hline \multirow{12}{*}{ rs72525532 } & \multirow{7}{*}{-} & hsa-mir-7113-3p & \multirow{12}{*}{ PolymiRTS Database, MirSNP, TargetScan, and miRanda } \\
\hline & & hsa-mir-2682-3p & \\
\hline & & hsa-mir-4287 & \\
\hline & & hsa-mir-4469 & \\
\hline & & hsa-mir-4685-3p & \\
\hline & & hsa-mir-6781-3p & \\
\hline & & hsa-mir-6867-3p & \\
\hline & \multirow{5}{*}{ GA } & hsa-miR-3667-3p & \\
\hline & & hsa-mir-4297 & \\
\hline & & hsa-mir-4691-5p & \\
\hline & & hsa-mir-6749-3p & \\
\hline & & hsa-mir-6792-3p & \\
\hline \multirow{5}{*}{ rs188133936 } & \multirow{2}{*}{ C } & hsa-mir-324-5p & \multirow{5}{*}{$\begin{array}{l}\text { TargetScan, miRanda, PolymiRTS Database, } \\
\text { and MirSNP }\end{array}$} \\
\hline & & hsa-miR-5700 & \\
\hline & & hsa-miR-2054 & \\
\hline & $\mathrm{T}$ & hsa-miR-374c-5p & \\
\hline & & hsa-mir-655-3p & \\
\hline \multirow{9}{*}{ rs45596738/rs397897431 } & & hsa-miR-1470 & \multirow{9}{*}{ PolymiRTS Database, MirSNP, TargetScan, and miRanda } \\
\hline & & hsa-mir-4287 & \\
\hline & & hsa-mir-4469 & \\
\hline & - & hsa-mir-4667-3p & \\
\hline & & hsa-mir-4685-3p & \\
\hline & & hsa-mir-6867-3p & \\
\hline & & hsa-mir-7113-3p & \\
\hline & CA & hsa-miR-6845-3p & \\
\hline & GA & hsa-mir-7110-3p & \\
\hline \multirow{7}{*}{ rs148759216 } & & hsa-mir-2682-3p & \multirow{7}{*}{ PolymiRTS Database, MirSNP, TargetScan, and miRanda } \\
\hline & - & hsa-mir-6781-3p & \\
\hline & \multirow{5}{*}{ AG } & hsa-mir-3183 & \\
\hline & & hsa-mir-4723-3p & \\
\hline & & hsa-mir-6769b-3p & \\
\hline & & hsa-mir-6845-3p & \\
\hline & & hsa-mir-7110-3p & \\
\hline
\end{tabular}

Table 2. Basic characteristics of participants in the case-control study

\begin{tabular}{|c|c|c|c|c|c|}
\hline & \multicolumn{2}{|c|}{ Case Group $(n=57)$} & \multicolumn{2}{|c|}{ Control Group $(n=63)$} & \multirow{2}{*}{$P$ Value } \\
\hline & Mean & SEM & Mean & SEM & \\
\hline Age (y) & 12.35 & 0.25 & 13.39 & 0.38 & 0.03 \\
\hline Boys/girls & $25 / 32$ & & $26 / 33$ & & 0.49 \\
\hline $\mathrm{BMI}\left(\mathrm{kg} / \mathrm{m}^{2}\right)$ & 26.68 & 0.52 & 18.31 & 1.06 & $<0.001$ \\
\hline TG (mg/dL) & 110.98 & 6.73 & 73.46 & 3.22 & $<0.001$ \\
\hline TC (mg/dL) & 161.86 & 4.02 & 141.61 & 4.27 & 0.005 \\
\hline $\mathrm{HDL}-\mathrm{C}(\mathrm{mg} / \mathrm{dL})$ & 43.25 & 0.69 & 49.03 & 1.23 & $<0.001$ \\
\hline LDL-C (mg/dL) & 89.68 & 2.77 & 76.14 & 1.99 & $<0.001$ \\
\hline
\end{tabular}

BMI: body mass index; TG: triglyceride; TC: total cholesterol; HDL-C: high-density lipoprotein cholesterol; LDL-C: low-density lipoprotein cholesterol; SEM:

standard error of mean. Values are expressed as mean \pm SEM.

individuals are shown in Table 3. The differences in the distribution of APOA5 genotypes in rs72525532 were statistically significant between case and control subjects. In addition, the frequency of AA insertion genotype was significantly higher in the Met $S$ group as compared to the control group $(P=0.012)$. The data demonstrated that the frequencies of AA insertion genotype were $1.7 \%$ and $12.28 \%$ in control and case groups, and the frequencies of no insertion genotype were $98.3 \%$ and $87.72 \%$ in control and case groups, respectively. The comparison of the laboratory parameters of the case and control subjects according to different $A P O A 5$ genotypes is summarized in Table 4. These data showed a significant difference between different genotype groups regarding TG and HDL-C levels, whereas no significant associations were observed between this variant and the other components of MetS.

On the other hand, TG levels were significantly higher 
Table 3. Genotype Frequencies of rs72525532 in Case and Control Groups

\begin{tabular}{|c|c|c|c|c|c|c|}
\hline \multirow{2}{*}{ Group } & \multirow{2}{*}{ Total N } & \multicolumn{2}{|c|}{ No. of Insertion } & \multicolumn{2}{|c|}{$\mathrm{A} / \mathrm{A}$} & \multirow{2}{*}{$P$ Value } \\
\hline & & No. & $\%$ & No. & $\%$ & \\
\hline Case & 57 & 50 & 87.72 & 7 & 12.28 & \multirow{2}{*}{0.012} \\
\hline Control & 59 & 58 & 98.3 & 1 & 1.7 & \\
\hline
\end{tabular}

among the control samples in AA insertion genotype group compared to no insertion genotype groups $(P<0.0001)$, whereas there was a borderline significant difference between the two genotype groups $(P=0.07)$ in MetS participants. Furthermore, HDL-C levels were lower among subjects with no insertion genotype in the control groups compared to AA insertion genotype groups $(P=0.05)$. The results of logistic regression analysis (Table 5) revealed that the AA insertion genotype was significantly associated with MetS risk [OR (95\% CI) = 8.12 (0.96668.27), $P=0.05$ ], while this association was insignificant after the adjustment for age [OR $(95 \% \mathrm{CI})=5.66(0.629$ 46.357), $P=0.124]$.

\section{Discussion}

The current study, to the best of our knowledge, was the first research that explored the associations of five polymorphisms of apolipoprotein A5 (APOA5) gene with MetS and its various components in children and adolescents. MetS is associated with the incidence of CVDs and mortality (26-28). Generally, SNPs of APOA5 are correlated with dyslipidemia, including the increased plasma TG levels (29-31).

Our previous study evaluated the association of some APOA5 the 3' untranslated region (3'UTR) SNPs with MetS among Iranian population (32). According to one study, miR-TS-SNPs are associated with MetS risk (16), but no study has yet investigated five polymorphisms located in the APOA5 gene, including rs188133936, rs114627122, rs45596738, rs148759216, and rs72525532 regarding their association with MetS. The result of the sequencing indicated no variations in four APOA5 gene variants (i.e., rs188133936, rs114627122, rs45596738, and rs148759216); however, an insertion A was found at 3' UTR position 285-286 in rs72525532. In the dbSNP database, the rs72525532 variant was shown as insertion GA at 3' UTR positions 285-286. Further, the genotype distributions of rs72525532 variant were statistically different between the case and control groups. MetS participants showed higher frequencies of A insertion compared to control groups. As regards TG levels, variant rs72525532 demonstrated a significant difference between both genotype groups $(P<0.001)$ among the control subjects, while participants with MetS showed a borderline significant difference $(P=0.06)$. Therefore, based on the data, participants with AA insertion genotype in the control group had significantly higher levels of TG as compared to those with no insertion genotypes. Conversely, the subject with no insertion AA genotypes in the control group had a lower level of HDL-C compared with the subject with insertion AA genotypes. Meanwhile, there was no significant association between rs72525532 polymorphism and other MetS components, which included low-density lipoprotein- $C$ and body mass index. Our results revealed an association between insertion AA genotypes and a higher risk of MetS although this association was insignificant after adjustment for the age. In a study conducted on the Han Chinese population, Ye et al evaluated the correlation between eight miR-TSSNPs and the MetS risk and concluded that rs5999924 and rs5750146 in 3' UTR at APOL5 gene were related to MetS. They also found significant relationships between rs11724758 in 3' UTR at the FABP2 gene with MetS. The HDL-C level in the carriers of the AA genotype of

Table 4. Associations of clinical parameters with rs72525532 genotype according to case-control status

\begin{tabular}{|c|c|c|c|c|c|c|}
\hline & \multicolumn{3}{|c|}{ Case } & \multicolumn{3}{|c|}{ Control } \\
\hline & No of Insertion & AA & $P$ Value & No of Insertion & AA & $P$ Value \\
\hline Age $(y)$ & $12.56 \pm 0.28$ & $11.29 \pm 0.29$ & 0.05 & $13.27 \pm 0.40$ & 13 & 0.43 \\
\hline BMI (kg/m²) & $26.91 \pm 0.57$ & $24.60 \pm 0.61$ & 0.09 & - & - & - \\
\hline TG (mg/dL) & $114.76 \pm 7.35$ & $84 \pm 12.35$ & 0.07 & $71.76 \pm 2.78$ & 172 & 0.001 \\
\hline TC (mg/dL) & $163.18 \pm 4.25$ & $152.43 \pm 12.57$ & 0.19 & $141.10 \pm 4.29$ & 171 & 0.06 \\
\hline HDL-C (mg/dL) & $43.08 \pm 0.77$ & $44.43 \pm 0.99$ & 0.14 & $48.88 \pm 1.24$ & 58 & 0.05 \\
\hline LDL-C (mg/dL) & $90.10 \pm 2.91$ & $86.71 \pm 9.37$ & 0.35 & $76.10 \pm 2.03$ & 78 & 0.186 \\
\hline
\end{tabular}

BMI: body mass index; TG: triglyceride; TC: total cholesterol; HDL-C: high-density lipoprotein cholesterol; LDL-C: low-density lipoprotein cholesterol.

Table 5. The OR at $95 \% \mathrm{Cl}$ calculated by multiple logistic regression analysis models

\begin{tabular}{lccc}
\hline Genotype & Crude OR $(\mathbf{9 5} \% \mathbf{C I})$ & $\boldsymbol{P}$ Value & Adjusted OR (95\% CI) \\
\hline AA vs. No insertion & $8.12(0.966-68.27)$ & 0.054 & $5.66(0.629-46.357)$ \\
\hline
\end{tabular}

Adjusted for age; OR: odds ratio; $\mathrm{Cl}$ : Confidence intervals. 
rs1 1724758 variant was significantly higher than that of the non-carrier subjects while the level of TG and fasting blood sugar were lower in the carriers of AA genotype (16).

The current study only tested the associations of the SNPs among the Isfahan population, which is considered as the limitation of the study. However, we explored the association of the SNPs with the MetS for the first time among children and adolescents, which is the strong point of the present study.

\section{Conclusion}

This study was the first to evaluate the association between five SNPss located at apolipoprotein A5 3'UTR and MetS and its major components risk. Our results suggest that rs72525532 might be correlated with TG and HDL-C levels. Further studies including more diverse participants of the Iranian population are valuable to strengthen our results in the future.

Conflict of interests

None.

\section{Ethical considerations}

The Ethics Committee of Isfahan University of Medical Sciences approved the current study (under the ethics code of 293284). Oral assent and informed written consent were taken from the participants and their parents, respectively.

\section{Acknowledgments}

This study was funded in part by a grant from the Research Institute of Biotechnology, Shahrekord University, Shahrekord and Isfahan University of Medical Sciences, Isfahan, Iran. The ethical code and the date of the approval of the research project are 293284 and 2014.10.7, respectively.

\section{References}

1. Third Report of the National Cholesterol Education Program (NCEP) Expert Panel on Detection, Evaluation, and Treatment of High Blood Cholesterol in Adults (Adult Treatment Panel III) final report. Circulation. 2002;106(25):3143-421.

2. Haffner SM. The metabolic syndrome: inflammation, diabetes mellitus, and cardiovascular disease. Am J Cardiol. 2006;97(2a):3a-11a. doi: 10.1016/j.amjcard.2005.11.010.

3. Reaven GM. Banting lecture 1988. Role of insulin resistance in human disease. Diabetes. 1988;37(12):1595-607. doi: 10.2337/diab.37.12.1595.

4. Azizi F, Salehi P, Etemadi A, Zahedi-Asl S. Prevalence of metabolic syndrome in an urban population: Tehran Lipid and Glucose Study. Diabetes Res Clin Pract. 2003;61(1):29-37.

5. Zabetian A, Hadaegh F, Sarbakhsh P, Azizi F. Weight change and incident metabolic syndrome in Iranian men and women; a 3 year follow-up study. BMC Public Health. 2009;9:138. doi: 10.1186/1471-2458-9-138.

6. Alberti G, Zimmet P, Shaw J, Bloomgarden Z, Kaufman F, Silink M. Type 2 diabetes in the young: the evolving epidemic: the international diabetes federation consensus workshop. Diabetes Care. 2004;27(7):1798-811. doi: 10.2337/ diacare.27.7.1798.

7. Menown IB, Murtagh G, Maher V, Cooney MT, Graham IM, Tomkin G. Dyslipidemia therapy update: the importance of full lipid profile assessment. Adv Ther. 2009;26(7):711-8. doi: 10.1007/s12325-009-0052-3.
8. Tai ES, Ordovas JM. Clinical significance of apolipoprotein A5. Curr Opin Lipidol. 2008;19(4):349-54. doi: 10.1097/ MOL.0b013e328304b681.

9. Kluger M, Heeren J, Merkel M. Apoprotein A-V: an important regulator of triglyceride metabolism. J Inherit Metab Dis. 2008;31(2):281-8. doi: 10.1007/s10545-008-0863-4.

10. Merkel M, Heeren J. Give me A5 for lipoprotein hydrolysis! J Clin Invest. 2005;115(10):2694-6. doi: 10.1172/jci26712.

11. Heneghan HM, Miller N, Kerin MJ. Role of microRNAs in obesity and the metabolic syndrome. Obes Rev. 2010;11(5):354-61. doi: 10.1111/j.1467-789X.2009.00659.x.

12. Lin PC, LiuTC, Chang CC, Chen YH, Chang JG. High-resolution melting (HRM) analysis for the detection of single nucleotide polymorphisms in microRNA target sites. Clin Chim Acta. 2012;413(13-14):1092-7. doi: 10.1016/j.cca.2012.03.007.

13. Duan R, Pak C, Jin P. Single nucleotide polymorphism associated with mature miR-125a alters the processing of primiRNA. Hum Mol Genet. 2007;16(9):1124-31. doi: 10.1093/ hmg/ddm062.

14. Li L, Meng T, Jia Z, Zhu G, Shi B. Single nucleotide polymorphism associated with nonsyndromic cleft palate influences the processing of miR-140. Am J Med Genet A. 2010;152a(4):856-62. doi: 10.1002/ajmg.a.33236.

15. Ye Y, Wang KK, Gu J, Yang H, Lin J, Ajani JA, et al. Genetic variations in microRNA-related genes are novel susceptibility loci for esophageal cancer risk. Cancer Prev Res (Phila). 2008;1(6):460-9. doi: 10.1158/1940-6207.capr-08-0135.

16. Ye Q, Zhao X, Xu K, Li Q, Cheng J, Gao Y, et al. Polymorphisms in lipid metabolism related miRNA binding sites and risk of metabolic syndrome. Gene. 2013;528(2):132-8. doi: 10.1016/j.gene.2013.07.036.

17. Ziebarth JD, Bhattacharya A, Chen A, Cui Y. PolymiRTS Database 2.0: linking polymorphisms in microRNA target sites with human diseases and complex traits. Nucleic Acids Res. 2012;40(Database issue):D216-21. doi: 10.1093/nar/gkr1026.

18. Salehi S, Emadi-Baygi M, Rezaei M, Kelishadi R, Nikpour P. Identification of a new single-nucleotide polymorphism within the apolipoprotein A5 gene, which is associated with metabolic syndrome. Adv Biomed Res. 2017;6:24. doi: 10.4103/2277-9175.201688.

19. Alberti KG, Zimmet P, Shaw J. Metabolic syndrome-a new world-wide definition. A Consensus Statement from the International Diabetes Federation. Diabet Med. 2006;23(5):469-80. doi: 10.1111/j.1464-5491.2006.01858.x.

20. Gong J, Tong Y, Zhang HM, Guo AY. miRNASNP: a database of miRNA related SNPs and their effects on miRNA function. BMC Bioinformatics. 2012;13(Suppl 18):A2. doi: 10.1186/1471-2105-13-S18-A2.

21. Yan Cui. [PolymiRTS Database 3.0. http://compbio.uthsc.edu/ miRSNP/.

22. Liu C, Zhang F, Li T, Lu M, Wang L, Yue W, et al. MirSNP, a database of polymorphisms altering miRNA target sites, identifies miRNA-related SNPs in GWAS SNPs and eQTLs. BMC Genomics. 2012;13:661. doi: 10.1186/1471-2164-13661.

23. TargetScan human. http://www.targetscan.org/vert_72/.

24. miRWalk2.0: a comprehensive atlas of microRNA-target interactions. http://www.umm.uni-heidelberg.de/apps/zmf/ mirwalk.

25. microRNA.org - Targets and Expression. August $2010 \mathrm{http}: / /$ www.microrna.org/microrna/home.do.

26. Grundy SM, Brewer HB Jr, Cleeman Jl, Smith SC Jr, Lenfant C. Definition of metabolic syndrome: report of the National Heart, Lung, and Blood Institute/American Heart Association conference on scientific issues related to definition. 
Salehi et al

Arterioscler Thromb Vasc Biol. 2004;24(2):e13-8. doi: 10.1161/01.atv.0000111245.75752.c6

27. Isomaa B, Almgren P, Tuomi T, Forsén B, Lahti K, Nissen M, et al. Cardiovascular morbidity and mortality associated with the metabolic syndrome. Diabetes Care. 2001;24(4):683-9. doi: 10.2337/diacare.24.4.683.

28. Hu G, Qiao Q, Tuomilehto J, Balkau B, Borch-Johnsen $\mathrm{K}$, Pyorala K. Prevalence of the metabolic syndrome and its relation to all-cause and cardiovascular mortality in nondiabetic European men and women. Arch Intern Med. 2004;164(10):1066-76. doi: 10.1001/archinte.164.10.1066.

29. Povel CM, Boer JM, Reiling E, Feskens EJ. Genetic variants and the metabolic syndrome: a systematic review. Obes Rev. 2011;12(11):952-67. doi: 10.1111/j.1467-
789X.2011.00907.x.

30. Maász A, Kisfali P, Horvatovich K, Mohás M, Markó L, Csöngei V, et al. Apolipoprotein A5 T-1131C variant confers risk for metabolic syndrome. Pathol Oncol Res. 2007;13(3):243-7. doi: Paor.2007.13.3.0243.

31. Melegh BI, Duga B, Sümegi K, Kisfali P, Maász A, Komlósi K, et al. Mutations of the apolipoprotein A5 gene with inherited hypertriglyceridaemia: review of the current literature. Curr Med Chem. 2012;19(36):6163-70.

32. Salehi S, Emadi-Baygi M, Rezaei M, Kelishadi R, Nikpour P. Lack of evidence of the role of APOA5 3'UTR polymorphisms in Iranian children and adolescents with metabolic syndrome. Diabetes Metab J. 2018;42(1):74-81. doi: 10.4093/ dmj.2018.42.1.74. 